\title{
Metallicities for 13 nearby open clusters from high-resolution spectroscopy of dwarf and giant stars ${ }^{\star}$
}

\section{Stellar metallicity, stellar mass, and giant planets}

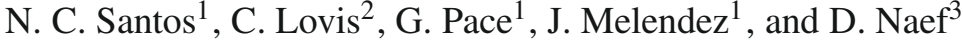 \\ ${ }^{1}$ Centro de Astrofísica, Universidade do Porto, Rua das Estrelas, 4150-762 Porto, Portugal \\ e-mail: nuno@astro.up.pt \\ 2 Observatoire de Genève, $51 \mathrm{ch}$. des Maillettes, 1290 Sauverny, Switzerland \\ 3 European Southern Observatory, Casilla 19001, Santiago 19, Chile
}

Received 6 October 2008 / Accepted 14 November 2008

\begin{abstract}
We present a study of accurate stellar parameters and iron abundances for 39 giants and 16 dwarfs in the 13 open clusters IC 2714 , IC 4651, IC 4756, NGC 2360, NGC 2423, NGC 2447 (M 93), NGC 2539, NGC 2682 (M 67), NGC 3114, NGC 3680, NGC 4349, NGC 5822, NGC 6633. The analysis was done using a set of high-resolution and high- $S / N$ spectra obtained with the UVES spectrograph (VLT). These clusters are currently being searched for planets using precise radial velocities. For all the clusters, the derived average metallicities are close to solar. Interestingly, the values derived seem to depend on the line-list used. This dependence and its implications for the study of chemical abundances in giants stars are discussed. We show that a careful choice of the lines may be crucial for the derivation of metallicities for giant stars on the same metallicity scale as those derived for dwarfs. Finally, we discuss the implications of the derived abundances for the metallicity- and mass-giant planet correlation. We conclude that a good knowledge of the two parameters is necessary to correctly disentangle their influence on the formation of giant planets.
\end{abstract}

Key words. stars: planetary systems: formation - stars: abundances - stars: fundamental parameters - techniques: spectroscopic Galaxy: open clusters and associations: general

\section{Introduction}

Precise spectroscopic studies of solar-type dwarfs with giant planets have shown that the frequency of giant planets is a strong function of the stellar metallicity. It seems easier to find a giant planet around a metal-rich star than around a metal-poor object (Gonzalez et al. 2001; Santos et al. 2001, 2004, 2005; Reid 2002; Fischer \& Valenti 2005) ${ }^{1}$. This observation has often been interpreted as being due to a higher probability of forming a giant planet in a metal-rich disk (e.g. Ida \& Lin 2004; Mordasini et al. 2007).

Interestingly, it has been pointed out that this metallicitygiant planet correlation may not hold for intermediate mass stars hosting giant planets (Pasquini et al. 2007). Pasquini et al. interpret this observation as due to the deeper convective envelopes of giant stars, which could have washed out the previously existing metallicity enhancement due to the accretion of planetary material. Although not fully accepted (Hekker \& Meléndez 2007), if confirmed this lack of correlation could alternatively be hinting that stellar mass strongly influences the planet formation process.

A few studies found evidence that the fraction of stars harboring planets is indeed an increasing function of stellar mass (Lovis \& Mayor 2007; Johnson et al. 2007a). For instance, the

\footnotetext{
* Based on observations collected at the La Silla Parana Observatory, ESO (Chile) with the UVES spectrograph at the 8.2-m Kueyen telescope, under programs 079.C-0131 and 66.D-0457.

${ }^{1}$ For a recent general review of the properties of the known exoplanets see Udry \& Santos (2007).
}

frequency of giant planets orbiting (low-mass) M-dwarfs is considerably lower than the one found for FGK dwarfs (Bonfils et al. 2005; Endl et al. 2006). This result is expected from the models of planetary formation (Laughlin et al. 2004; Ida \& Lin 2005; Kennedy \& Kenyon 2008), although a consensus does not exist regarding this point (Kornet et al. 2005; Boss 2006).

One way to address these issues is to search for planets around (intermediate-mass) giant stars. The search for planets around such objects is motivated by some of the limitations of the radial-velocity technique. Although very effective to search for planets around solar-type stars, for main-sequence higher mass objects this method is usually not very efficient. This is due to the lack of spectral information (lines), together with the usually high rotation velocities of higher mass dwarfs.

Intermediate mass stars evolving out of the main sequence, however, become cooler and (usually) slower rotators. This fact makes of them good targets for radial-velocity searches, and give the possibility to use such techniques to probe the frequency of planets around higher mass stars $\left(1.5-4 M_{\odot}\right)$.

A few teams started radial-velocity programs to search for planets around sub-giant or giant field stars. As a consequence, a few giant planet candidates have been announced around these kind of objects (e.g. Frink et al. 2002; Sato et al. 2003; Setiawan et al. 2005; Hatzes et al. 2006; Niedzielski et al. 2007; Johnson et al. 2007b). Unfortunately, it is not easy to derive the mass for a field giant star, making it difficult to study the "stellar massfrequency of planets" relation for these objects.

One way around this difficulty is to search for planets orbiting giants in galactic open clusters. Three giant planets were 
detected so far by such surveys, namely orbiting NGC 2423 No3, NGC 4349 No127 (Lovis \& Mayor 2007), and $\epsilon$ Tau (Sato et al. 2007). In this case, the derivation of the mass of the turnoff stars in the cluster gives us immediate information about the mass of the clump giants. Furthermore, if we observe clusters with different ages we will be studying stars within a whole range of stellar masses.

In this paper we derive atmospheric parameters and chemical abundances for giant and dwarfs stars in 13 clusters that are being monitored with radial-velocities to search for giant planets (Lovis \& Mayor 2007). In Sects. 2 and 3 we present our sample and the observations. In Sect. 4 we present the analysis of the data and compare the metallicities derived from giants and dwarf stars for the same cluster. In Sects. 5 and 6 we present the results, discussing in particular their implications for the metallicity- and mass-giant planet correlations. We conclude in Sect 7.

\section{Sample}

For statistical and redundancy reasons, we chose to observe 3 clump giants in each of the 13 clusters studied in this paper. The clusters were taken from the sample of Lovis \& Mayor (2007): IC 2714, IC 4651, IC 4756, NGC 2360, NGC 2423, NGC 2447 (M93), NGC 2539, NGC 2682 (M 67), NGC 3114, NGC 3680, NGC 4349, NGC 5822, NGC 6633. We point the reader to this paper for more details on the sample clusters.

The choice of the specific targets to observe was based on CORALIE and HARPS radial-velocity data collected by these authors in their planet-search program. The final list of targets (Table 1) includes either stars hosting a candidate giant planet or stars that are stable in radial-velocity within the measurement errors. This allows us to exclude spectroscopic binary stars from our sample.

The majority of the studies about the metallicity-giant planet connection are based on the analysis of field dwarfs (e.g. Santos et al. 2004, 2005; Fischer \& Valenti 2005; Sousa et al. 2008). It is thus important to check that the metallicities derived for the giants match those derived for the dwarfs of the same cluster. With this goal, a number of G-dwarfs in 4 of the clusters mentioned above were also observed.

For NGC 2447, three dwarfs (Table 1) were chosen based on the radial-velocity measurements obtained in a separate planetsearch project (ESO programs 076.C-0525 and 078.C-0481). This allowed us to exclude known spectroscopic binaries, nonmembers, as well as stars with high projected rotational velocities. Details on the observations and catalog where these stars were selected will be described in detail in Naef et al. (in prep.).

For the dwarfs in IC 4651, NGC 2682, and NGC 3680, the spectra used were presented in Pace et al. (2008) as part of a study of chemical abundances in 4 different open clusters. As we will see below, the metallicity values derived here are in perfect agreement with those derived by these authors (using the same spectra but a different methodology).

Finally, given the very good agreement between the values determined by Pace et al. (2008) and the ones presented here for the same stars, the average metallicity values presented by Pace et al. 2009 (in preparation) for dwarfs in IC 4756 and NGC 5822 were also added to the list.

\section{Observations}

Spectra for all the 39 giants in the 13 clusters, as well as for the three G-dwarfs in NGC 2447, were obtained using the
Table 1. Target list of UVES-VLT program 079.C-0131.

\begin{tabular}{|c|c|c|c|}
\hline Star & $\alpha(2000.0)$ & $\delta(2000.0)$ & V \\
\hline \multicolumn{4}{|l|}{ Giant stars } \\
\hline NGC 2360 No7 & 07:18:19.01 & $-15: 34: 59.4$ & 11.10 \\
\hline NGC 2360 No79 & 07:17:16.91 & $-15: 38: 40.7$ & 11.29 \\
\hline NGC 2360 No85 & $07: 17: 32.56$ & $-15: 38: 59.4$ & 11.44 \\
\hline NGC 2423 No3 & 07:37:09.24 & $-13: 54: 24.0$ & 10.08 \\
\hline NGC 2423 No20 & $07: 37: 11.53$ & $-13: 55: 44.1$ & 11.08 \\
\hline NGC 2423 No240 & $07: 37: 46.60$ & $-13: 49: 44.3$ & 10.67 \\
\hline NGC 2447 No28 & $07: 44: 50.25$ & $-23: 52: 27.1$ & 9.85 \\
\hline NGC 2447 No34 & 07:44:33.66 & $-23: 51: 42.2$ & 10.12 \\
\hline NGC 2447 No41 & 25.73 & $-23: 49: 53.0$ & 10.03 \\
\hline NGC 2539 No229 & 08:10:33.80 & $-12: 51: 48.9$ & 11.17 \\
\hline NGC 2539 No346 & & -12 & 10.92 \\
\hline NGC $2539 \mathrm{No}$ & & -12 & 11.03 \\
\hline NGC 2682 No164 & 08: & +1 & 10.63 \\
\hline NGC 2682 No266 & 08: & +1 & 10.55 \\
\hline NGC 2682 No286 & 08: & +1 & 10.41 \\
\hline NGC 3114 No13 & $10: 0$ & -60 & 9.09 \\
\hline NGC 3114 No181 & 10:03:51.49 & $-60: 03: 10.2$ & 8.30 \\
\hline NGC 3114 No273 & 10:02:41.26 & $-60: 29: 03.3$ & 9.78 \\
\hline IC 2714 No53 & 11:18:15.89 & $-62: 42: 04.0$ & 11.52 \\
\hline IC 2714 No87 & $11: 17: 46.85$ & $-62: 36: 49.5$ & 11.39 \\
\hline IC 2714 No110 & $11: 17: 30.57$ & $-62: 42: 27.5$ & 11.60 \\
\hline NGC 3680 No13 & $11: 2$ & $-43: 14: 24.0$ & 10.82 \\
\hline NGC 3680 No26 & 11: & -43 & 10. \\
\hline NGC $3680 \mathrm{~N}$ & 1 & 2.5 & 10.92 \\
\hline NGC 4349 No5 & 52 & & 11.51 \\
\hline NGC 4349 No127 & $12: 2$ & 1.7 & 10.83 \\
\hline NGC 4349 No168 & $12: 2$ & 3.5 & 11.48 \\
\hline NGC 5822 No102 & 42 & .7 & 10.82 \\
\hline NGC 5822 No2 & 15 & .9 & 10.81 \\
\hline NGC 5822 No438 & $15: 05: 31.14$ & $-54: 26: 20.0$ & 10.94 \\
\hline IC 4651 No7646 & $17: 24: 41.53$ & $-49: 59: 06.4$ & 10.31 \\
\hline IC 4651 No9122 & $17: 24: 50.09$ & $-49: 56: 56.1$ & 10.86 \\
\hline IC 4651 No10393 & $17: 24: 57.75$ & $-50: 01: 32.9$ & 10.64 \\
\hline NGC 6633 No100 & $18: 27: 54.73$ & $+06: 36: 00.3$ & 8.30 \\
\hline NGC 6633 No119 & $18: 28: 17.64$ & $+06: 46: 00.1$ & 8.95 \\
\hline NGC 6633 No126 & $18: 28: 22.98$ & $+06: 42: 29.3$ & 8.77 \\
\hline IC 4756 No38 & $18: 37: 05.21$ & $+05: 17: 31.6$ & 9.76 \\
\hline IC 4756 No42 & 18:37:20.77 & $+05: 53: 43.1$ & 9.46 \\
\hline IC 4756 No125 & 18:39:17.88 & $+05: 13: 48.8$ & 9.29 \\
\hline \multicolumn{4}{|l|}{ Dwarf stars } \\
\hline NGC 244713625 & 07:44:10.23 & $-24: 05: 46.1$ & 15.36 \\
\hline NGC 244713815 & 07:44:11.43 & $-23: 53: 28.3$ & 14.88 \\
\hline NGC 244718606 & 07:44:41.97 & $-23: 59: 14.1$ & 15.00 \\
\hline
\end{tabular}

UVES spectrograph (VLT Kueyen telescope), between April and October 2007 (ESO programs 079.C-0131A and B). For consistency, we opted for the same instrument configuration for all the observations. A slit width of 0.9 arcsec was used, providing a spectral resolution $R=\lambda / \Delta \lambda \sim 50000$. The observations were done using the RED580 mode, and the resulting spectra cover the wavelength domain between 4780 and $6805 \AA$, with a gap between 5730 and $5835 \AA$ (corresponding to the gap in the CCD mosaic). The $S / N$ of the final spectra is between 200 and 300 for the giants, and $\sim 100$ for the dwarfs.

For some of the stars, attention was given to the orientation of the UVES slit due to the relative crowdedness of the fields.

As mentioned above, for IC 4651, NGC 2682, and NGC 3680, the spectra used are presented in Pace et al. (2008). These data were also obtained with the UVES spectrograph (ESO run 66.D-0457), and have a spectral resolution of 100000 (a slit width of 0.4 arcsec was used). All spectra cover the same wavelength interval as the data described above. The final 
spectra have a typical $S / N$ of $\sim 80$. We point the reader to these authors for more details about this set of data.

Finally, the spectroscopic parameters derived by Pace et al. (2009, in preparation) and presented in this paper for dwarfs in IC 4756 and NGC 5822, were also obtained from the analysis of UVES data. In this case, the spectra have a resolution of $\sim 100000$, and $S / N \sim 100$. These spectra cover the same wavelength domain as the remaining data.

\section{Analysis}

Stellar atmospheric parameters and iron abundances were derived in LTE using the 2002 version of the code MOOG (Sneden $1973)^{2}$ and a grid of Kurucz Atlas plane-parallel model atmospheres (Kurucz 1993). The final parameters were obtained imposing excitation and ionization equilibrium to a set of $\mathrm{Fe}$ I and Fe II lines, following the basic prescription described in Santos et al. (2004).

The determination of the uncertainties in the derived parameters also follow the same prescription as in Santos et al. (2004). The error bars only reflect internal uncertainties. We conservatively use the rms around the average abundance given by $\mathrm{Fe}$ I lines, and not the error on the average, to derive the error in $[\mathrm{Fe} / \mathrm{H}]$. This means that the tabled uncertainties are likely upper limits.

Line equivalent widths (EW) for the iron lines were measured using the automatic ARES code (Sousa et al. 2007, 2008) ${ }^{3}$. Star-by-star visual inspection of sample EW measurements was done in order to verify if the ARES parameters used were adequate for the resolution and $S / N$ of the data.

Slightly different procedures were then adopted for the spectroscopic analysis of the dwarfs and giants. In the former case, stellar parameters were derived using the line-list described in Sousa et al. (2008, hereafter S08). This list, composed of 263 Fe I and $36 \mathrm{Fe}$ II lines in the optical domain has shown to give excellent results for the analysis of dwarf stars. For the giants, further to this we also derived the stellar parameters using the linelist provided by Hekker \& Meléndez (2007, hereafter HM07). Although much smaller ( $20 \mathrm{Fe}$ I and 6 Fe II lines), the lines in this list were carefully chosen for the analysis of giant stars, avoiding line-blending from CN lines (Meléndez \& Barbuy 1999).

For the three giants in NGC 4651, the small EW interval of the Fe I lines measured using the smaller line-list of HM07 did not allow a consistent determination of the microturbulence. We have thus decided to use, only in this case, microturbulence values derived using the empirical formula used by these authors (based on a relation between microturbulence and effective temperature).

The derived stellar parameters and iron abundances for all the stars analyzed in this paper are listed in Tables 2-4.

\subsection{Comparison with other works}

The large majority of the clusters studied here do not have any previous metallicity estimate derived from high-resolution spectroscopy.

Pace et al. (2008) compiled a long list of chemical abundances for open clusters studied in the literature using high resolution spectroscopy. A simple comparison of their Tables 3 and 9 for the three clusters in common (namely IC 4651, NGC 2682

\footnotetext{
2 http://verdi.as.utexas. edu/moog.html

3 This software can be downloaded at http://www.astro.up.pt/ $\sim$ sousasag/ares/
}

(M 67), and NGC 3680) show a very good agreement, not only with the values derived by these authors but also with other literature values.

We note that in this paper we analyzed the same spectra as Pace et al. (2008) for the dwarfs in these three clusters, although we used a different procedure to derive the stellar parameters. Pace et al. find average $[\mathrm{Fe} / \mathrm{H}]$ values of $+0.12 \pm 0.05$, $+0.03 \pm 0.04$, and $-0.04 \pm 0.03$ for IC 4651, NGC 2682 (M 67), and NGC 3680, respectively. From the same spectra we derive average metallicities of $+0.15 \pm 0.02,+0.01 \pm 0.04$, and $-0.03 \pm 0.01$.

Chen et al. (2003a) compiled metallicity estimates (mostly photometric) for more than 150 open clusters. Except for NGC 2447, all the 13 clusters studied here have metallicity values listed by these authors. A comparison of their $[\mathrm{Fe} / \mathrm{H}]$ values with those derived in our paper reveal a reasonable good agreement. The average difference between our metallicity values and those in Chen et al. is only -0.03 dex (median of -0.04), our determinations having a higher average value (considering the estimates based on the S08 line-list). The dispersion is, however, relatively high, with the differences ranging between +0.05 and -0.15 dex.

\subsection{Comparing giants and dwarfs}

The analysis of both dwarfs and giant stars in 6 of the studied clusters (IC 4651, IC 4756, NGC 2447, NGC 2682, NGC 3680, and NGC 5822) allows us to verify if the metallicity scale of the two classes of objects is the same.

In Tables 5 and 6 we list the average values for the metallicities derived for each cluster, using the giant and dwarf stars, respectively. For the giants, both determinations are used, using the S08 and HM07 line-lists (Cols. 2 and 4 in Table 5). In general terms, a good agreement is seen. Values determined using giant stars and the S08 line-list show a star-to-star dispersion that is clearly smaller than the one obtained using the HM07 line-list. This result is somewhat expected given the much higher number of iron lines used in the former case.

In Fig. 1 we compare the average metallicity values for the 6 clusters derived using the dwarfs stars with the same values derived using giants, both with the S08 (left panel) and HM07 (right panel) line-lists. As mentioned above, in both cases we can see a general agreement. However, it is clear from the plots that while in the latter case there is no evidence of a clear difference on the metallicity scale for giants and dwarfs, in the former case this does not seem to be true. A linear fit to the points in the left panel of Fig. 1 holds a relation $\langle[\mathrm{Fe} / \mathrm{H}]\rangle_{\text {dwarfs }}=1.97\langle[\mathrm{Fe} / \mathrm{H}]\rangle_{\mathrm{S} 08}-0.03$, with a very small dispersion of 0.004 dex. Interestingly, for near solar metallicities all estimates almost perfectly agree.

This result suggests that the metallicities derived for giant stars using the HM07 line-list are closer to the expected value, supposing that dwarfs provide a correct metallicity scale. We remember that the line-list compiled by HM07 was carefully chosen for the analysis of giant stars, while the former was optimized for the study of solar-type dwarfs.

Given the smaller dispersion of the $[\mathrm{Fe} / \mathrm{H}]$ values derived for giant stars using the S08 line-list, it may be interesting, however, to use these values after correcting for the trend mentioned above. The corrected values are listed in Table 5 (Col. 3, $\left.\langle[\mathrm{Fe} / \mathrm{H}]\rangle_{\mathrm{S} 08}^{\mathrm{c}}\right)$.

We decided to adopt these values for the rest of the paper. After correcting the S08 values for the relation mentioned above, a 1 to 1 relation is also found when comparing with the $[\mathrm{Fe} / \mathrm{H}]$ 
Table 2. Stellar parameters derived for the giants stars using the line-list of Sousa et al. (2008, S08). See text for more details.

\begin{tabular}{|c|c|c|c|c|c|c|}
\hline 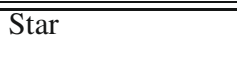 & $\begin{array}{l}T_{\text {eff }} \\
{[\mathrm{K}]}\end{array}$ & $\begin{array}{l}\log g_{\text {spec }} \\
{\left[\mathrm{cm} \mathrm{s}^{-2}\right]}\end{array}$ & $\begin{array}{c}\xi_{\mathrm{t}} \\
{\left[\mathrm{km} \mathrm{s}^{-1}\right]}\end{array}$ & 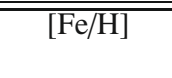 & 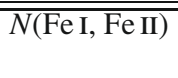 & 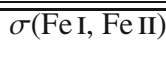 \\
\hline IC 2714 No53 & $5211 \pm 36$ & $2.89 \pm 0.36$ & $1.64 \pm 0.04$ & $0.02 \pm 0.11$ & 181,25 & $0.11,0.16$ \\
\hline IC 2714 No87 & $5253 \pm 38$ & $3.03 \pm 0.31$ & $1.69 \pm 0.04$ & $0.03 \pm 0.12$ & 179,24 & $0.11,0.14$ \\
\hline IC 2714 No110 & $5128 \pm 31$ & $2.92 \pm 0.27$ & $1.56 \pm 0.03$ & $0.02 \pm 0.10$ & 183,24 & $0.10,0.13$ \\
\hline IC 4651 No7646 & $4974 \pm 34$ & $2.80 \pm 0.28$ & $1.54 \pm 0.03$ & $0.09 \pm 0.10$ & 182,23 & $0.10,0.14$ \\
\hline IC 4651 No9122 & $4787 \pm 43$ & $2.82 \pm 0.35$ & $1.52 \pm 0.05$ & $0.09 \pm 0.12$ & 177,24 & $0.12,0.19$ \\
\hline IC 4651 No10393 & $4903 \pm 43$ & $2.79 \pm 0.30$ & $1.33 \pm 0.04$ & $0.10 \pm 0.12$ & 180,25 & $0.12,0.15$ \\
\hline IC 4756 No38 & $5225 \pm 26$ & $3.16 \pm 0.22$ & $1.40 \pm 0.02$ & $0.05 \pm 0.08$ & 179,24 & $0.08,0.10$ \\
\hline IC 4756 No42 & $5240 \pm 26$ & $3.14 \pm 0.22$ & $1.39 \pm 0.02$ & $0.01 \pm 0.08$ & 181,24 & $0.08,0.10$ \\
\hline IC 4756 No125 & $5207 \pm 26$ & $3.06 \pm 0.26$ & $1.47 \pm 0.02$ & $0.02 \pm 0.08$ & 181,24 & $0.08,0.12$ \\
\hline NGC 2360 No7 & $5182 \pm 22$ & $3.09 \pm 0.25$ & $1.45 \pm 0.02$ & $-0.00 \pm 0.07$ & 181,25 & $0.07,0.12$ \\
\hline NGC 2360 No79 & $5201 \pm 25$ & $3.07 \pm 0.30$ & $1.46 \pm 0.02$ & $0.01 \pm 0.08$ & 180,25 & $0.08,0.14$ \\
\hline NGC 2360 No85 & $5195 \pm 26$ & $3.21 \pm 0.29$ & $1.40 \pm 0.02$ & $-0.00 \pm 0.08$ & 183,25 & $0.08,0.14$ \\
\hline NGC 2423 No3 & $4703 \pm 49$ & $2.48 \pm 0.40$ & $1.67 \pm 0.05$ & $-0.00 \pm 0.15$ & 180,24 & $0.15,0.21$ \\
\hline NGC 2423 No20 & $5122 \pm 33$ & $3.09 \pm 0.20$ & $1.43 \pm 0.03$ & $0.12 \pm 0.09$ & 180,24 & $0.09,0.10$ \\
\hline NGC 2423 No240 & $5104 \pm 35$ & $2.91 \pm 0.20$ & $1.51 \pm 0.03$ & $0.09 \pm 0.10$ & 183,25 & $0.10,0.10$ \\
\hline NGC 2447 No28 & $5125 \pm 23$ & $2.77 \pm 0.22$ & $1.63 \pm 0.02$ & $-0.06 \pm 0.07$ & 174,23 & $0.07,0.10$ \\
\hline NGC 2447 No34 & $5222 \pm 24$ & $2.95 \pm 0.16$ & $1.62 \pm 0.02$ & $-0.01 \pm 0.08$ & 174,24 & $0.08,0.08$ \\
\hline NGC 2447 No41 & $5190 \pm 26$ & $2.91 \pm 0.23$ & $1.62 \pm 0.02$ & $-0.03 \pm 0.08$ & 177,25 & $0.08,0.11$ \\
\hline NGC 2539 No229 & $5145 \pm 34$ & $3.03 \pm 0.20$ & $1.49 \pm 0.03$ & $0.09 \pm 0.10$ & 183,24 & $0.10,0.10$ \\
\hline NGC 2539 No346 & $5149 \pm 33$ & $2.97 \pm 0.22$ & $1.56 \pm 0.03$ & $0.05 \pm 0.10$ & 182,25 & $0.10,0.10$ \\
\hline NGC 2539 No502 & $5211 \pm 34$ & $2.86 \pm 0.47$ & $1.60 \pm 0.03$ & $0.10 \pm 0.10$ & 180,25 & $0.10,0.21$ \\
\hline NGC 2682 No164 & $4812 \pm 42$ & $2.73 \pm 0.21$ & $1.57 \pm 0.04$ & $0.03 \pm 0.12$ & 180,22 & $0.12,0.11$ \\
\hline NGC 2682 No266 & $4862 \pm 37$ & $2.76 \pm 0.23$ & $1.59 \pm 0.03$ & $0.01 \pm 0.11$ & 179,23 & $0.11,0.12$ \\
\hline NGC 2682 No286 & $4817 \pm 38$ & $2.69 \pm 0.23$ & $1.59 \pm 0.04$ & $0.01 \pm 0.11$ & 176,23 & $0.11,0.12$ \\
\hline NGC 3114 No13 & $4985 \pm 34$ & $2.77 \pm 0.18$ & $1.57 \pm 0.03$ & $0.01 \pm 0.10$ & 181,24 & $0.10,0.09$ \\
\hline NGC 3114 No181 & $4561 \pm 45$ & $1.92 \pm 0.32$ & $2.02 \pm 0.04$ & $-0.11 \pm 0.13$ & 165,24 & $0.13,0.17$ \\
\hline NGC 3114 No273 & $5305 \pm 24$ & $3.09 \pm 0.20$ & $1.57 \pm 0.02$ & $0.07 \pm 0.07$ & 183,25 & $0.07,0.09$ \\
\hline NGC 3680 No13 & $4781 \pm 41$ & $2.79 \pm 0.19$ & $1.48 \pm 0.04$ & $-0.01 \pm 0.12$ & 181,21 & $0.12,0.10$ \\
\hline NGC 3680 No26 & $4758 \pm 50$ & $2.70 \pm 0.23$ & $1.40 \pm 0.05$ & $-0.01 \pm 0.14$ & 177,23 & $0.14,0.12$ \\
\hline NGC 3680 No41 & $4823 \pm 39$ & $2.85 \pm 0.25$ & $1.45 \pm 0.04$ & $0.01 \pm 0.12$ & 180,23 & $0.12,0.13$ \\
\hline NGC 4349 No5 & $5186 \pm 42$ & $2.72 \pm 0.45$ & $1.90 \pm 0.04$ & $-0.00 \pm 0.14$ & 180,25 & $0.14,0.21$ \\
\hline NGC 4349 No127 & $4569 \pm 69$ & $2.08 \pm 0.35$ & $1.93 \pm 0.06$ & $-0.13 \pm 0.18$ & 172,23 & $0.17,0.19$ \\
\hline NGC 4349 No168 & $5154 \pm 34$ & $2.56 \pm 0.34$ & $1.88 \pm 0.03$ & $-0.03 \pm 0.12$ & 180,25 & $0.12,0.16$ \\
\hline NGC 5822 No102 & $5253 \pm 28$ & $3.17 \pm 0.39$ & $1.44 \pm 0.03$ & $-0.00 \pm 0.08$ & 178,24 & $0.08,0.17$ \\
\hline NGC 5822 No224 & $5214 \pm 28$ & $3.14 \pm 0.20$ & $1.41 \pm 0.03$ & $0.06 \pm 0.08$ & 184,25 & $0.08,0.09$ \\
\hline NGC 5822 No438 & $5208 \pm 25$ & $3.16 \pm 0.19$ & $1.39 \pm 0.02$ & $0.06 \pm 0.08$ & 180,25 & $0.08,0.09$ \\
\hline NGC 6633 No100 & $5118 \pm 29$ & $2.83 \pm 0.25$ & $1.65 \pm 0.03$ & $0.04 \pm 0.10$ & 183,26 & $0.10,0.12$ \\
\hline NGC 6633 No119 & $5275 \pm 30$ & $3.10 \pm 0.24$ & $1.43 \pm 0.03$ & $0.04 \pm 0.09$ & 184,24 & $0.09,0.11$ \\
\hline NGC 6633 No126 & $5251 \pm 29$ & $3.10 \pm 0.29$ & $1.50 \pm 0.03$ & $0.06 \pm 0.09$ & 183,24 & $0.09,0.13$ \\
\hline
\end{tabular}

derived using the HM07 line-list. We caution, however, that the use of only 6 clusters may limit the validity of this correction. More data may be needed to confirm which of the line-lists provides the best metallicity determinations for the giant stars.

We tested the two line-list used, deriving the solar parameters and iron abundance using an available solar "Ganymede" spectrum taken with the HARPS spectrograph ${ }^{4}$. In both cases, EW were measured with ARES using the parameters set as discussed in S08. As expected, in the former case the solar parameters almost perfectly match the expected values $\left(T_{\mathrm{eff}}, \log g, \xi_{\mathrm{t}},[\mathrm{Fe} / \mathrm{H}]\right.$ of $5765 \pm 28 \mathrm{~K}, 4.46 \pm 0.11,0.97 \pm 0.04$, and $-0.01 \pm 0.05$, respectively). Using the HM07 line-list we obtain a slightly different solution $(5777 \pm 38 \mathrm{~K}, 4.63 \pm 0.12$, $1.03 \pm 0.06,+0.08 \pm 0.05)$.

\subsection{The metallicity scale for giants}

The bias mentioned above implies that using the metallicities derived for giants with the S08 line-list we would obtain a significant narrower $[\mathrm{Fe} / \mathrm{H}]$ distribution. In other words, we would

\footnotetext{
4 http://www.eso.org/sci/facilities/lasilla/ instruments/harps/inst/monitoring/sun.html
}

overestimate the $[\mathrm{Fe} / \mathrm{H}]$ values for metal-poor objects, while strongly underestimating the metallicities for any putative high $[\mathrm{Fe} / \mathrm{H}]$ giants.

This issue may be related to the fact that (cool) giant stars have higher macroturbulence velocities (Gray 1992), and that thousands of molecular lines $(\mathrm{CN}, \mathrm{C} 2, \mathrm{CH}, \mathrm{MgH})$ contribute to the optical spectra of giants (Coelho et al. 2005). Both effects lead to stronger line-blending, implying a wrong estimate of the continuum position when measuring line-EWs. This effect should be more important for higher metallicity objects, explaining the stronger difference observed for the most metal-rich stars.

This effect may be enhanced if the spectral resolution is not sufficiently high. Using different resolution degraded solar spectra and the ARES code with fixed input parameters (as an approximation), Sousa et al. (2007) suggest that for spectral resolutions below $\sim 50000$, the EW estimates may tend to systematically decrease as a function of decreasing resolution. In our case, all spectra have a resolution above $\sim 50000$, likely limiting the importance of this problem. We cannot exclude, however, that this effect is more important for cool giant stars, for which line crowdedness is stronger. 
Table 3. Stellar parameters derived for the giants stars using the line-list of Hekker \& Meléndez (2007, HM07). See text for more details.

\begin{tabular}{|c|c|c|c|c|c|c|}
\hline Star & $\begin{array}{l}T_{\text {eff }} \\
{[\mathrm{K}]}\end{array}$ & $\begin{array}{l}\log g_{\text {spec }} \\
{\left[\mathrm{cm} \mathrm{s}^{-2}\right]}\end{array}$ & $\begin{array}{c}\xi_{\mathrm{t}} \\
{\left[\mathrm{km} \mathrm{s}^{-1}\right]}\end{array}$ & $\overline{[\mathrm{Fe} / \mathrm{H}]}$ & $N(N(\mathrm{Fe}$ I, Fe II $)$ & 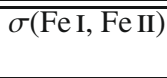 \\
\hline IC 2714 No53 & $5045 \pm 65$ & $2.75 \pm 0.16$ & $1.47 \pm 0.11$ & $-0.05 \pm 0.10$ & 16,5 & $0.08,0.07$ \\
\hline IC 2714 No87 & $5029 \pm 70$ & $2.62 \pm 0.27$ & $1.44 \pm 0.11$ & $-0.06 \pm 0.11$ & 16,6 & $0.08,0.13$ \\
\hline IC 2714 No110 & $5017 \pm 66$ & $2.85 \pm 0.18$ & $1.43 \pm 0.10$ & $0.01 \pm 0.10$ & 16,6 & $0.08,0.08$ \\
\hline IC 4651 No7646 & $4847 \pm 84$ & $2.61 \pm 0.18$ & $1.23 \dagger$ & $0.20 \pm 0.10$ & 16,6 & $0.09,0.06$ \\
\hline IC 4651 No9122 & $4676 \pm 83$ & $2.88 \pm 0.19$ & $1.32 \dagger$ & $0.23 \pm 0.09$ & 16,6 & $0.09,0.05$ \\
\hline IC 4651 No10393 & $4784 \pm 76$ & $2.70 \pm 0.24$ & $1.26 \dagger$ & $0.12 \pm 0.10$ & 16,6 & $0.09,0.11$ \\
\hline IC 4756 No38 & $5151 \pm 73$ & $3.16 \pm 0.17$ & $1.22 \pm 0.11$ & $0.08 \pm 0.11$ & 16,6 & $0.08,0.07$ \\
\hline IC 4756 No42 & $5217 \pm 89$ & $3.21 \pm 0.17$ & $1.15 \pm 0.13$ & $0.10 \pm 0.11$ & 15,6 & $0.08,0.06$ \\
\hline IC 4756 No125 & $5146 \pm 82$ & $3.11 \pm 0.18$ & $1.31 \pm 0.13$ & $0.07 \pm 0.11$ & 15,6 & $0.08,0.07$ \\
\hline NGC 2360 No7 & $5074 \pm 67$ & $3.05 \pm 0.15$ & $1.48 \pm 0.12$ & $-0.04 \pm 0.10$ & 16,6 & $0.08,0.06$ \\
\hline NGC 2360 No79 & $5122 \pm 69$ & $3.16 \pm 0.15$ & $1.43 \pm 0.11$ & $0.03 \pm 0.10$ & 15,6 & $0.07,0.06$ \\
\hline NGC 2360 No85 & $5091 \pm 69$ & $3.17 \pm 0.14$ & $1.37 \pm 0.11$ & $-0.01 \pm 0.10$ & 16,6 & $0.07,0.05$ \\
\hline NGC 2423 No3 & $4578 \pm 96$ & $2.49 \pm 0.22$ & $1.60 \pm 0.10$ & $0.00 \pm 0.11$ & 16,6 & $0.09,0.06$ \\
\hline NGC 2423 No20 & $5034 \pm 75$ & $3.13 \pm 0.14$ & $1.39 \pm 0.10$ & $0.12 \pm 0.09$ & 15,6 & $0.07,0.04$ \\
\hline NGC 2423 No240 & $5030 \pm 75$ & $2.96 \pm 0.13$ & $1.50 \pm 0.11$ & $0.08 \pm 0.11$ & 16,6 & $0.08,0.04$ \\
\hline NGC 2447 No28 & $5038 \pm 100$ & $2.76 \pm 0.15$ & $1.74 \pm 0.16$ & $-0.12 \pm 0.12$ & 12,6 & $0.08,0.04$ \\
\hline NGC 2447 No34 & $5076 \pm 75$ & $2.88 \pm 0.10$ & $1.65 \pm 0.13$ & $-0.07 \pm 0.11$ & 13,6 & $0.08,0.02$ \\
\hline NGC 2447 No41 & $5064 \pm 84$ & $2.93 \pm 0.12$ & $1.70 \pm 0.14$ & $-0.10 \pm 0.12$ & 13,6 & $0.08,0.03$ \\
\hline NGC 2539 No229 & $5067 \pm 71$ & $3.08 \pm 0.13$ & $1.47 \pm 0.11$ & $0.11 \pm 0.10$ & 16,6 & $0.08,0.04$ \\
\hline NGC 2539 No346 & $5066 \pm 72$ & $2.93 \pm 0.13$ & $1.38 \pm 0.10$ & $0.08 \pm 0.11$ & 16,6 & $0.08,0.04$ \\
\hline NGC 2539 No502 & $5061 \pm 81$ & $2.93 \pm 0.13$ & $1.45 \pm 0.11$ & $0.07 \pm 0.11$ & 15,5 & $0.08,0.04$ \\
\hline NGC 2682 No164 & $4659 \pm 93$ & $2.53 \pm 0.20$ & $1.51 \pm 0.10$ & $-0.02 \pm 0.11$ & 16,6 & $0.09,0.05$ \\
\hline NGC 2682 No266 & $4747 \pm 68$ & $2.62 \pm 0.15$ & $1.48 \pm 0.09$ & $-0.00 \pm 0.10$ & 16,6 & $0.08,0.05$ \\
\hline NGC 2682 No286 & $4721 \pm 75$ & $2.68 \pm 0.20$ & $1.55 \pm 0.09$ & $0.02 \pm 0.09$ & 16,6 & $0.07,0.08$ \\
\hline NGC 3114 No13 & $4871 \pm 60$ & $2.60 \pm 0.14$ & $1.45 \pm 0.08$ & $0.01 \pm 0.08$ & 15,6 & $0.06,0.06$ \\
\hline NGC 3114 No181 & $4384 \pm 147$ & $1.65 \pm 0.36$ & $2.01 \pm 0.15$ & $-0.23 \pm 0.17$ & 16,6 & $0.14,0.08$ \\
\hline NGC 3114 No273 & $5193 \pm 66$ & $3.00 \pm 0.16$ & $1.50 \pm 0.11$ & $0.07 \pm 0.10$ & 15,6 & $0.07,0.07$ \\
\hline NGC 3680 No13 & $4657 \pm 74$ & $2.68 \pm 0.17$ & $1.38 \pm 0.07$ & $-0.01 \pm 0.08$ & 16,6 & $0.06,0.05$ \\
\hline NGC 3680 No26 & $4649 \pm 125$ & $2.68 \pm 0.28$ & $1.41 \pm 0.14$ & $-0.02 \pm 0.14$ & 15,6 & $0.11,0.08$ \\
\hline NGC 3680 No41 & $4676 \pm 75$ & $2.70 \pm 0.17$ & $1.38 \pm 0.08$ & $-0.03 \pm 0.09$ & 16,6 & $0.07,0.05$ \\
\hline NGC 4349 No5 & $4970 \pm 78$ & $2.54 \pm 0.17$ & $1.69 \pm 0.13$ & $-0.09 \pm 0.12$ & 16,5 & $0.09,0.07$ \\
\hline NGC 4349 No127 & $4394 \pm 105$ & $1.91 \pm 0.31$ & $1.81 \pm 0.10$ & $-0.14 \pm 0.12$ & 16,6 & $0.10,0.13$ \\
\hline NGC 4349 No168 & $5092 \pm 54$ & $2.76 \pm 0.16$ & $1.75 \pm 0.09$ & $-0.00 \pm 0.08$ & 15,5 & $0.06,0.07$ \\
\hline NGC 5822 No102 & $5170 \pm 42$ & $3.20 \pm 0.13$ & $1.18 \pm 0.07$ & $0.05 \pm 0.06$ & 13,6 & $0.04,0.06$ \\
\hline NGC 5822 No224 & $5237 \pm 65$ & $3.37 \pm 0.12$ & $1.15 \pm 0.08$ & $0.22 \pm 0.08$ & 14,6 & $0.06,0.04$ \\
\hline NGC 5822 No438 & $5148 \pm 62$ & $3.21 \pm 0.11$ & $1.13 \pm 0.08$ & $0.18 \pm 0.08$ & 16,6 & $0.06,0.04$ \\
\hline NGC 6633 No100 & $4979 \pm 72$ & $2.75 \pm 0.12$ & $1.58 \pm 0.10$ & $0.00 \pm 0.10$ & 16,6 & $0.08,0.03$ \\
\hline NGC 6633 No119 & $5163 \pm 81$ & $2.97 \pm 0.12$ & $1.25 \pm 0.13$ & $0.01 \pm 0.12$ & 16,6 & $0.09,0.04$ \\
\hline NGC 6633 No126 & $5124 \pm 69$ & $2.92 \pm 0.18$ & $1.45 \pm 0.12$ & $0.00 \pm 0.11$ & 16,6 & $0.08,0.08$ \\
\hline
\end{tabular}

$\dagger$ Fixed using a $T_{\text {eff }}-\xi_{\text {t }}$ calibration (see text).

The problem of the metallicity scale for giants stars was already discussed by Taylor \& Croxall (2005). These authors further pointed out that no giant stars are observed in the solarneighborhood having a metallicity above $\sim 0.2 \mathrm{dex}$. Whether this observation is real or due to some bias in the spectroscopic analysis should be addressed. Although more data is needed, the results presented above may support the second hypothesis. A careful choice of the lines used for the analysis of giants stars is certainly needed.

Finally, the observed bias may have important implications for the study of stellar populations, like the galactic bulge, where dwarfs are too faint to be observed. Indeed, the few studies of the chemical abundances of dwarfs in the bulge have suggested that these may be more metal rich than previously estimated from the analysis of giant stars (e.g. Cohen et al. 2008).

\section{The metallicity of nearby open clusters}

In Fig. 2 we present the metallicity distribution of the 13 clusters studied in this paper. For this plot we used the average metallicities listed in Table 5, derived using the S08 line-list and corrected using the relation discussed in Sect. 4.2. A similar result would be obtained if we were plotting the metallicity values deriving using the HM07 line-list.

The metallicity distribution of the 13 clusters studied here has a mean value of +0.02 dex, somehow higher than the value observed for average field dwarfs $(-0.09$ dex $)$, and for the open clusters from Table 1 of Chen et al. (2003b) ( -0.07 dex). The rms of the cluster distribution is also smaller ( $0.08 \mathrm{dex}$, compared to 0.24 dex for the field dwarfs and 0.23 of the Chen et al. sample). The average difference with respect to this latter sample is probably not significant, given the small number of clusters analyzed here, and the possible existence of small systematic errors in the metallicities derived using different methods (photometry vs. spectroscopy).

\section{Giants with giant planets}

Two of the giants studied in this work are known to be orbited by giant planets: NGC 2423 No3, NGC 4349 No127 (Lovis $\&$ Mayor 2007). The two clusters have average metallicities of $+0.14 \pm 0.06$ and $-0.12 \pm 0.06$, respectively (using the corrected 
Table 4. Stellar parameters derived for the dwarf stars. See text for more details.

\begin{tabular}{|c|c|c|c|c|c|c|}
\hline Star & $\begin{array}{l}T_{\text {eff }} \\
{[\mathrm{K}]}\end{array}$ & $\begin{array}{l}\log g_{\text {spec }} \\
{\left[\mathrm{cm} \mathrm{s}^{-2}\right]}\end{array}$ & $\begin{array}{c}\xi_{\mathrm{t}} \\
{\left[\mathrm{km} \mathrm{s}^{-1}\right]}\end{array}$ & {$[\mathrm{Fe} / \mathrm{H}]$} & $\mathrm{N}(\mathrm{Fe}$ I, Fe II $)$ & $\overline{\sigma(\mathrm{Fe}} \mathrm{I}, \mathrm{Fe}$ II $)$ \\
\hline IC 4651 AMC1109 & $6075 \pm 32$ & $4.54 \pm 0.22$ & $1.14 \pm 0.04$ & $0.15 \pm 0.06$ & 168,24 & $0.06,0.08$ \\
\hline IC 4651 AMC2207 & $6139 \pm 34$ & $4.58 \pm 0.16$ & $1.17 \pm 0.04$ & $0.17 \pm 0.06$ & 166,22 & $0.06,0.06$ \\
\hline IC 4651 AMC4220 & $5793 \pm 45$ & $4.40 \pm 0.26$ & $0.97 \pm 0.06$ & $0.15 \pm 0.10$ & 169,23 & $0.09,0.10$ \\
\hline IC 4651 AMC4226 & $5862 \pm 59$ & $4.31 \pm 0.46$ & $0.89 \pm 0.07$ & $0.13 \pm 0.13$ & 172,23 & $0.12,0.17$ \\
\hline IC 4651 Eggen45 & $6280 \pm 44$ & $4.40 \pm 0.16$ & $1.30 \pm 0.05$ & $0.11 \pm 0.10$ & 167,22 & $0.09,0.06$ \\
\hline NGC 244713625 & $5595 \pm 30$ & $4.45 \pm 0.19$ & $1.16 \pm 0.04$ & $-0.12 \pm 0.06$ & 170,21 & $0.06,0.08$ \\
\hline NGC 244713815 & $5920 \pm 28$ & $4.59 \pm 0.10$ & $1.25 \pm 0.04$ & $-0.09 \pm 0.05$ & 164,21 & $0.05,0.04$ \\
\hline NGC 244718606 & $5795 \pm 33$ & $4.47 \pm 0.20$ & $1.16 \pm 0.05$ & $-0.11 \pm 0.07$ & 167,22 & $0.07,0.08$ \\
\hline NGC 2682 Sanders746 & $5703 \pm 30$ & $4.46 \pm 0.15$ & $0.97 \pm 0.04$ & $-0.07 \pm 0.06$ & 168,22 & $0.06,0.06$ \\
\hline NGC 2682 Sanders 1048 & $5915 \pm 35$ & $4.48 \pm 0.32$ & $0.96 \pm 0.05$ & $0.07 \pm 0.07$ & 170,24 & $0.07,0.12$ \\
\hline NGC 2682 Sanders1092 & $6074 \pm 29$ & $4.39 \pm 0.08$ & $1.35 \pm 0.03$ & $0.04 \pm 0.06$ & 167,21 & $0.06,0.03$ \\
\hline NGC 2682 Sanders 1255 & $5892 \pm 23$ & $4.47 \pm 0.21$ & $1.23 \pm 0.03$ & $0.01 \pm 0.05$ & 165,26 & $0.05,0.08$ \\
\hline NGC 2682 Sanders1283 & $6028 \pm 29$ & $4.42 \pm 0.13$ & $1.15 \pm 0.03$ & $0.00 \pm 0.06$ & 168,22 & $0.06,0.05$ \\
\hline NGC 2682 Sanders 1287 & $6111 \pm 42$ & $4.47 \pm 0.26$ & $1.09 \pm 0.05$ & $0.01 \pm 0.09$ & 161,21 & $0.08,0.09$ \\
\hline NGC 3680 AHTC1009 & $5932 \pm 28$ & $4.47 \pm 0.16$ & $1.06 \pm 0.04$ & $-0.03 \pm 0.06$ & 167,24 & $0.06,0.06$ \\
\hline NGC 3680 Eggen70 & $6134 \pm 42$ & $4.54 \pm 0.22$ & $1.22 \pm 0.06$ & $-0.04 \pm 0.09$ & 166,22 & $0.08,0.08$ \\
\hline
\end{tabular}

Table 5. Weighted average metallicities of the 3 giant stars in each of the 13 clusters. $\langle[\mathrm{Fe} / \mathrm{H}]\rangle_{\mathrm{S} 08},\langle[\mathrm{Fe} / \mathrm{H}]\rangle_{\mathrm{S} 08}^{\mathrm{c}}$, and $\langle[\mathrm{Fe} / \mathrm{H}]\rangle_{\mathrm{HM} 07}$ represent the average values of the metallicities derived using the Sousa et al. (2008) line-list without and with the correction discussed in Sect. 4.2, and the average of the values derived using the line-list of Hekker \& Meléndez (2007).

\begin{tabular}{lccc}
\hline \hline Cluster & $\langle[\mathrm{Fe} / \mathrm{H}]\rangle_{\mathrm{S} 08}$ & $\langle[\mathrm{Fe} / \mathrm{H}]\rangle_{\mathrm{S} 08}^{\mathrm{c}}$ & $\langle[\mathrm{Fe} / \mathrm{H}]\rangle_{\mathrm{HM} 07}$ \\
\hline IC 2714 & $0.02 \pm 0.01$ & $0.01 \pm 0.01$ & $-0.03 \pm 0.04$ \\
IC 4651 & $0.09 \pm 0.01$ & $0.15 \pm 0.01$ & $0.19 \pm 0.06$ \\
IC 4756 & $0.02 \pm 0.02$ & $0.02 \pm 0.02$ & $0.08 \pm 0.01$ \\
NGC 2360 & $0.00 \pm 0.01$ & $-0.03 \pm 0.01$ & $-0.01 \pm 0.03$ \\
NGC 2423 & $0.09 \pm 0.06$ & $0.14 \pm 0.06$ & $0.07 \pm 0.06$ \\
NGC 2447 & $-0.03 \pm 0.03$ & $-0.10 \pm 0.03$ & $-0.10 \pm 0.03$ \\
NGC 2539 & $0.08 \pm 0.03$ & $0.13 \pm 0.03$ & $0.09 \pm 0.02$ \\
NGC 2682 & $0.02 \pm 0.01$ & $0.00 \pm 0.01$ & $0.00 \pm 0.02$ \\
NGC 3114 & $0.02 \pm 0.09$ & $0.02 \pm 0.09$ & $0.00 \pm 0.12$ \\
NGC 3680 & $-0.00 \pm 0.01$ & $-0.04 \pm 0.01$ & $-0.02 \pm 0.01$ \\
NGC 4349 & $-0.04 \pm 0.06$ & $-0.12 \pm 0.06$ & $-0.06 \pm 0.08$ \\
NGC 5822 & $0.04 \pm 0.04$ & $0.05 \pm 0.04$ & $0.12 \pm 0.10$ \\
NGC 6633 & $0.04 \pm 0.01$ & $0.06 \pm 0.01$ & $0.00 \pm 0.00$ \\
\hline
\end{tabular}

Table 6. Weighted average metallicities of the dwarf stars in each of the studied clusters.

\begin{tabular}{lccc}
\hline \hline Cluster & $\langle[\mathrm{Fe} / \mathrm{H}]\rangle_{\text {dwarfs }}$ & $N_{\text {stars }}$ & Source \\
\hline IC4651 & $0.15 \pm 0.02$ & 5 & $(1)$ \\
IC4756 & $0.01 \pm 0.04$ & 3 & $(2)$ \\
NGC 2447 & $-0.10 \pm 0.02$ & 3 & $(1)$ \\
NGC 2682 & $0.01 \pm 0.04$ & 6 & $(1)$ \\
NGC 3680 & $-0.03 \pm 0.00$ & 2 & $(1)$ \\
NGC 5822 & $0.05 \pm 0.03$ & 2 & $(2)$ \\
\hline
\end{tabular}

(1) This paper; (2) Pace et al. (in prep.).

values in Table 5). These two clusters have the second highest, and the lowest metallicity values among the 13 studied clusters, respectively for NGC 2423 and NGC 4349.

The small $[\mathrm{Fe} / \mathrm{H}]$ interval and the low number of planets discovered so far in this sample prevents us from taking any clear conclusions regarding the metallicity-giant planet connection. All the clusters span a $[\mathrm{Fe} / \mathrm{H}]$ domain where a large number of planets have been found orbiting dwarf field stars (e.g. Santos et al. 2005; Fischer \& Valenti 2005; Sousa et al. 2008).
There is currently some debate about whether the metallicity-giant planet correlation that is found for field dwarfs (e.g. Santos et al. 2004; Fischer \& Valenti 2005) is also valid for giant stars (Pasquini et al. 2007; Hekker \& Meléndez 2007). Pasquini et al. suggested that this trend in indeed not found for field giants. In face of our present results, their conclusions should be partially taken with care. It could be that their metallicities for metal-rich giants have been underestimated, leading to a spurious lack of very metal-rich planet hosting giants.

Furthermore, we note that the determination of accurate stellar masses for field giants is problematic. In this sense, it is interesting to see that although NGC 4349 is among the most metalpoor clusters analyzed here, the mass of its giants $\left(3.9 \pm 0.3 M_{\odot}\right.$ - Lovis \& Mayor 2007) is one of the highest among the sample (see Table 1 of that paper). In comparison, the giants in the moderately metal-rich cluster NGC 2423 are less massive $\left(2.4 \pm 0.2 M_{\odot}\right)$. If we consider that stellar mass positively influences the planet formation efficiency, this may hint that stellar mass may be compensating for the lower metallicity in the case of NGC 4349, justifying the discovery of a giant planet among one of its giants.

It is curious to see that both NGC 2423 No3 and NGC 4349 No127 are amongst the coolest stars studied in this paper, and the coolest and more metal-poor objects among the three stars analyzed in their respective clusters (although the metallicities all agree within the error-bars). We cannot exclude that the lower metallicities reflect the existence of systematic errors in the analysis of lower-temperature stars, as blending by molecular lines will be more severe for these objects (Coelho et al. 2005). A look at Tables 2 and 3 show that there is a small tendency for lower temperature stars in a given cluster to present also lower metallicity values. This possible trend should be studied using more giant stars covering a wide range of temperatures in each of the clusters.

\section{Concluding remarks}

We presented a study of iron abundances in 39 giants and 16 dwarfs in 13 open clusters using high resolution spectroscopy. All these clusters are part of a survey for giant planets orbiting intermediate-mass giant stars.

The results show that all the clusters studied have $[\mathrm{Fe} / \mathrm{H}]$ values close to solar, with a small dispersion. Interestingly, the 

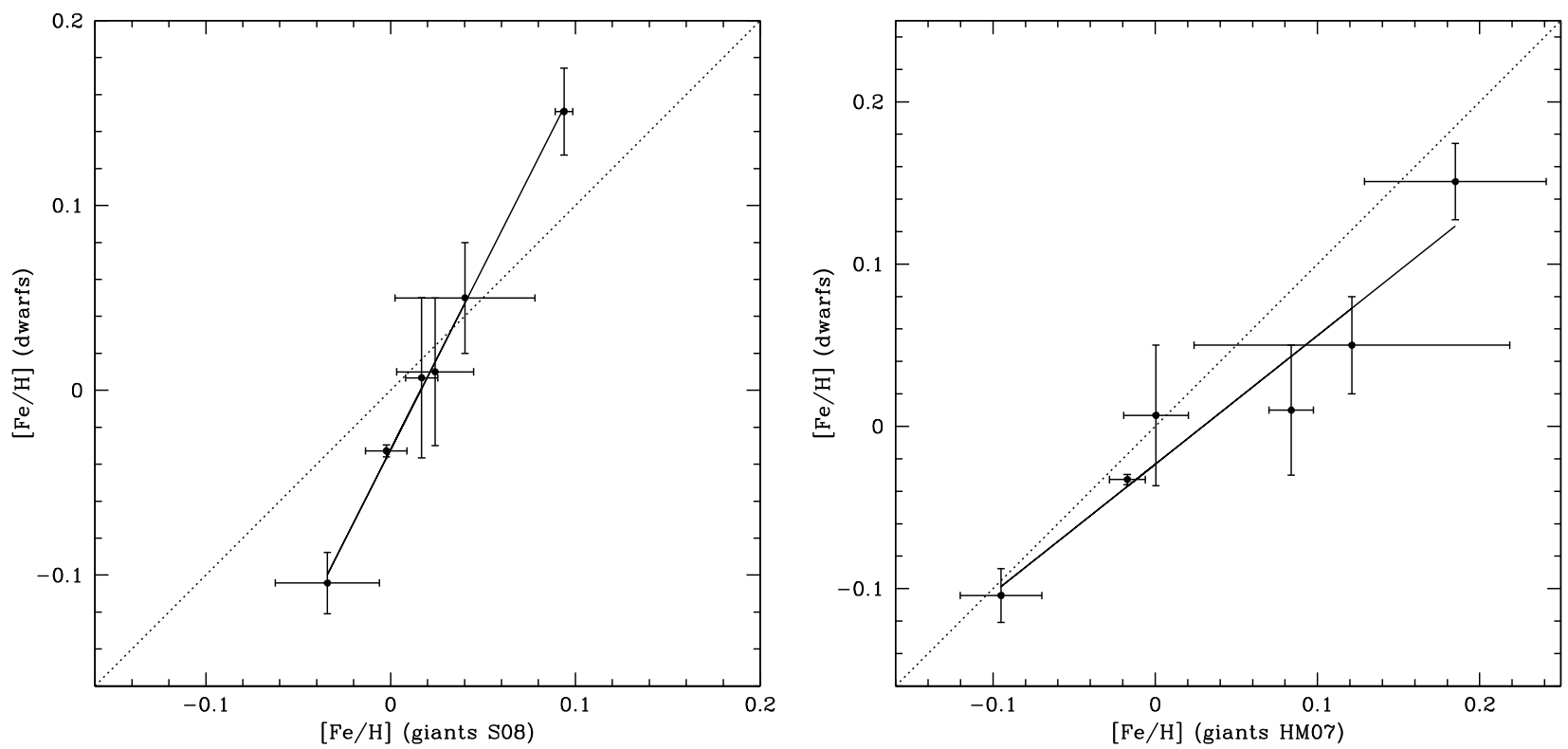

Fig. 1. Comparison of the average values for the metallicities derived from dwarfs and giants in the 6 clusters where analysis was done using both groups of stars. The dotted line represents the 1:1 relation, while the solid line represents a linear fit to the points. S08 and HM07 refer to the abundances derived using the line-lists of Sousa et al. (2008) and Hekker \& Meléndez (2007), respectively. The fit in the left panels holds the relation $\langle[\mathrm{Fe} / \mathrm{H}]\rangle_{\mathrm{dwarfs}}=1.97\langle[\mathrm{Fe} / \mathrm{H}]\rangle_{\mathrm{S} 08}-0.03$, with an rms of only 0.004 dex.

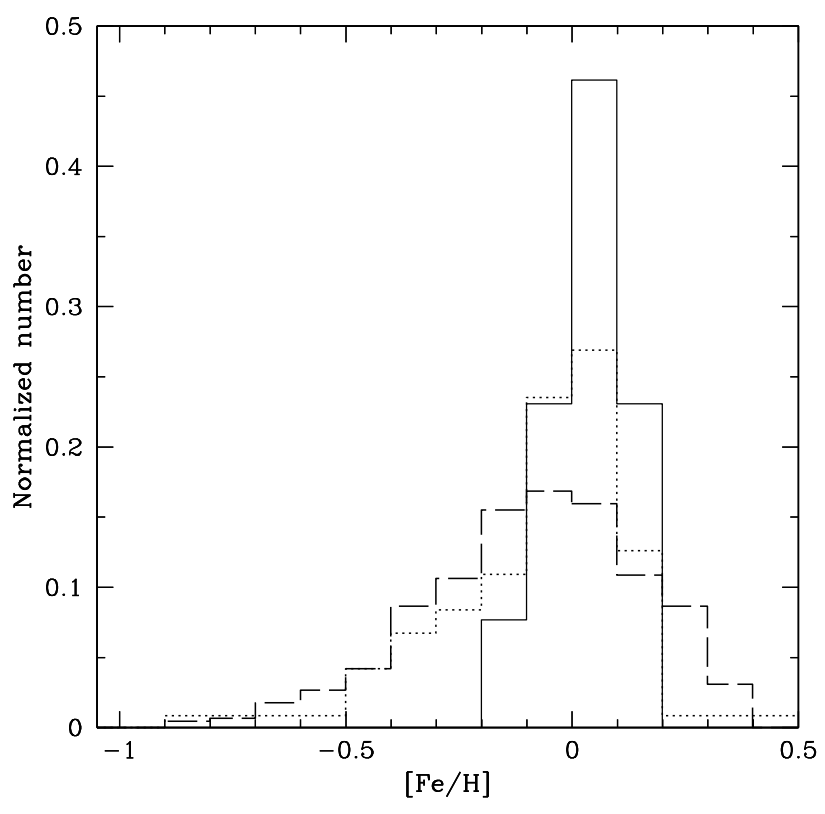

Fig. 2. Metallicity distribution of the average metallicities for the clusters studied in this work using the corrected S08 values listed in Table 5 (filled line), compared with the same distribution for 451 field dwarfs (dashed line) from (Sousa et al. 2008), and with the $[\mathrm{Fe} / \mathrm{H}]$ distribution of 119 open clusters (dotted line) described in Chen et al. (2003b).

metallicity values obtained for the giant stars depend on the line-list used to derive stellar parameters and iron abundances. When comparing with the dwarfs in our sample, we show that a careful choice of the lines used to derive parameters for giant stars may be necessary to avoid important biases on the metallicity scale for these objects. This result may have important implications for the study of chemical abundances in stellar populations using cool giant stars.

Two of the stars studied (in NGC 2423 and NGC 4349) are known to be orbited by long period giant planets. NGC 2423 is one of the most metal-rich clusters in our sample. Interestingly, the mass of the giant stars in the most metal-poor of these clusters (NGC 4349) is one of the highest among the 13 clusters. In the context of the metallicity- and stellar mass-giant planet correlation, this may be hinting that stellar mass may be compensating for the lower metallicity of this cluster. In any case, this result shows that to disentangle the metallicity and mass effects on the frequency of planets we need to have accurate values for both parameters.

Acknowledgements. N.C.S. and J.M. would like to thank the support from Fundação para a Ciência e a Tecnologia, Portugal, through programme Ciência 2007.

\section{References}

Bonfils, X., Forveille, T., Delfosse, X., et al. 2005, A\&A, 443, L15 Boss, A. P. 2006, ApJL, 644, L79

Chen, L., Hou, J. L., \& Wang, J. J. 2003a, AJ, 125, 1397

Chen, Y. Q., Zhao, G., Nissen, P. E., Bai, G. S., \& Qiu, H. M. 2003b, ApJ, 591, 925

Coelho, P., Barbuy, B., Meléndez, J., Schiavon, R. P., \& Castilho, B. V. 2005, A\&A, 443, 735

Cohen, J. G., Huang, W., Udalski, A., Gould, A., \& Johnson, J. A. 2008, ApJ, 682, 1029

Endl, M., Cochran, W. D., Kürster, M., et al. 2006, ApJ, 649, 436

Fischer, D. A., \& Valenti, J. 2005, ApJ, 622, 1102

Frink, S., Mitchell, D. S., Quirrenbach, A., et al. 2002, ApJ, 576, 478

Gonzalez, G., Laws, C., Tyagi, S., \& Reddy, B. E. 2001, AJ, 121, 432

Gray, D. F. 1992, The Observation and Analysis of Stellar Photospheres, ed.

D. F. Gray (Cambridge, UK: Cambridge University Press), 470

Hatzes, A. P., Cochran, W. D., Endl, M., et al. 2006, A\&A, 457, 335

Hekker, S., \& Meléndez, J. 2007, A\&A, 475, 1003

Ida, S., \& Lin, D. N. C. 2004, ApJ, 616, 567

Ida, S., \& Lin, D. N. C. 2005, ApJ, 626, 1045 
Johnson, J. A., Butler, R. P., Marcy, G. W., et al. 2007a, ApJ, 670, 833 Johnson, J. A., Fischer, D. A., Marcy, G. W., et al. 2007b, ApJ, 665, 785 Kennedy, G. M., \& Kenyon, S. J. 2008, ApJ, 673, 502

Kornet, K., Bodenheimer, P., Różyczka, M., \& Stepinski, T. F. 2005, A\&A, 430, 1133

Kurucz, R. 1993, ATLAS9 Stellar Atmosphere Programs and $2 \mathrm{~km} \mathrm{~s}^{-1}$ grid, Kurucz CD-ROM No. 13, Cambridge, Mass.: Smithsonian Astrophysical Observatory, 13

Laughlin, G., Bodenheimer, P., \& Adams, F. C. 2004, ApJ, 612, L73

Lovis, C., \& Mayor, M. 2007, A\&A, 472, 657

Meléndez, J., \& Barbuy, B. 1999, ApJS, 124, 527

Mordasini, C., Alibert, Y., Benz, W., \& Naef, D. 2007, ArXiv e-prints, 710

Niedzielski, A., Konacki, M., Wolszczan, A., et al. 2007, ApJ, 669, 1354

Pace, G., Pasquini, L., \& François, P. 2008, ArXiv e-prints, 806
Pasquini, L., Döllinger, M. P., Weiss, A., et al. 2007, A\&A, 473, 979 Reid, I. N. 2002, PASP, 114, 306

Santos, N. C., Israelian, G., \& Mayor, M. 2001, A\&A, 373, 1019

Santos, N. C., Israelian, G., \& Mayor, M. 2004, A\&A, 415, 1153

Santos, N. C., Israelian, G., Mayor, M., et al. 2005, A\&A, 437, 1127

Sato, B., Ando, H., Kambe, E., et al. 2003, ApJ, 597, L157

Sato, B., Izumiura, H., Toyota, E., et al. 2007, ApJ, 661, 527

Setiawan, J., Rodmann, J., da Silva, L., et al. 2005, A\&A, 437, L31

Sneden, C. 1973, Ph.D. Thesis, Univ. of Texas

Sousa, S. G., Santos, N. C., Israelian, G., Mayor, M., \& Monteiro, M. J. P. F. G. 2007, A\&A, 469, 783

Sousa, S. G., Santos, N. C., Mayor, M., et al. 2008, A\&A, 487, 373

Taylor, B. J., \& Croxall, K. 2005, MNRAS, 357, 967

Udry, S., \& Santos, N. 2007, ARA\&A, 45, 397 